\title{
Science beyond boundary: Are premature discoveries things of the past?
}

\begin{tabular}{|r|l|}
\hline Journal: & Genome \\
\hline Manuscript ID & gen-2016-0056 \\
\hline Manuscript Type: & Comment \\
\hline Date Submitted by the Author: & $10-$ Mar-2016 \\
\hline Complete List of Authors: & Singh, Rama; McMaster University \\
\hline Keyword: & $\begin{array}{l}\text { Science boundary, premature discovery, physics, paradigms, innovative } \\
\text { science }\end{array}$ \\
\hline & \\
\hline
\end{tabular}

SCHOLARONE $^{\text {Im }}$
Manuscripts 
Commentary

Science beyond boundary: Are premature discoveries things of the past?

\author{
Rama Singh \\ Department of Biology, McMaster University, Hamilton, Canada
}

Corresponding Author: Rama Singh singh@mcmaster.ca

Key words: $\quad$ science boundaries, premature discoveries, Mendel, McClintock, gender discrimination in science,

Number of words: $\quad 3,772$ 


\begin{abstract}
Mendel's name more than of any other draws our attention to the personal side in terms of success and failure in science. Mendel lived 19 years after presenting his research findings and died without receiving any recognition for his work. Are premature discoveries things of the past, you may ask? I review the material basis of science in terms of science boundary and field accessibility and analyze the possibility of premature discoveries in different fields of science such as, for example, physics and biology. I conclude that science has reached a stage where progress is being made mostly by pushing the boundary of the known from inside than by leaping across boundaries. As more researchers become engaged in science, and as more publications become open access, on-line and interactive, the probability of an important discovery remaining buried and going unrecognized would become exceedingly small. Of course, as examples from physics show, a new theory or an important idea can always lie low unrecognized until it becomes re-discovered and popularized by other researchers. Premature discoveries will become less likely but not forbidden.
\end{abstract}


One of the most remarkable things about biology is that the subject matter of our investigation lies all around us. You can pick any one of them at random, barring viruses, and use it to learn pretty much all you need to know about biology save evolution of higher intelligence and consciousness. The differences among higher organisms are icing on the cake as compared to the origin of life and the evolution of the basic structural plans. This is the reason behind using model organisms in biological investigations. This abundance of model systems, organisms bounded in space and time, is unique to biology and not available in any other field of science.

The 2015 marked the $150^{\text {th }}$ anniversary of the presentation of Mendel's paper, "Experiments on Plant Hybrids", before the Brno Society of Natural Science in 1865 which was published a year later in 1866 (Mendel 1866). The seminal results of Mendel's paper, based on eight years of work on the inheritance and stability of variation from generation to generation, went unappreciated until they were rediscovered by three scientists independently in 1900. Mendel died unrecognized in 1884. Nearly fifty years later Barbara McClintock started working on the extreme form of variation observed in seed coat color of corn and discovered mobile genetic elements. Her work was not recognized until much later when similar results were obtained with microorganisms. McClintock survived and received Nobel Prize in 1984. Mendel's and McClintock's works are said to be premature, before their time, as the scientific community was not ready to understand and appreciate the changing paradigms of science.

In an article written to mark Mendel's anniversary, I pointed out that imaginations have limits and unguided imaginations go nowhere (Singh 2015). Mendel's work has come under much criticism some of it unfairly though (for a recent analysis sees Hartl and Fairbanks 2007). Both Mendel's and McClintock's discoveries were supreme and yet both failed to draw attention of the scientists of the time. The question arises if science has advanced far enough that such premature discoveries are less likely to occur and are things of the past? In this review I summarize the nature of science boundaries and their degree of accessibility and show that in open or partly open fields, such as mathematics and physics, important ideas and theories can always lay aside until they become widely recognized and/or utilized; on the other hand, with the 
bounded nature of its subject matter (organisms in space and time) and their full accessibility there will be less and less chance of seeing premature discoveries in biology.

Major discoveries can gain recognition instantaneously or within a few years after their significance becomes known. A premature discovery is a major discovery that for any number of reasons did not get recognition in the first instance and it became eclipsed until it was rediscovered later. Such discoveries are said to be ahead of their time. The first discoverer may live long enough to see his/her discovery recognized as was the case with Barbara McClintock for her discovery of mobile genetic elements; or he or she may get due recognition posthumously as was the case with Gregor Mendel. Another group of premature discoveries are those which were not ignored but their full significance did not become clear until later. The latter definition may fit many cases of premature discoveries in physics more appropriately.

There are many reasons why a discovery may go unnoticed and to see that we will reflect on how discoveries are made, with a few examples here. I will follow this with the work of Mendel and McClintock and show how they both worked with the same black box (the heredity machinery) but worked with different kinds of inputs- outputs and obtained different results. This will be followed with a comparison of premature discoveries between physics and biology in an attempt to answer the question: "Are premature discoveries things of the past?"

\section{The material basis of science and science boundaries}

Scientific discoveries are shaped by three important factors, that is, beside social, political, and economic condition of the society: (1) the nature of the scientific discipline, e.g. discovery in biology are more based on instrumentation than, for example, in physics although particle physics has become hugely instrumental such as the use of mega cyclotron; (2) the historical development of previous work - this is true for all disciplines; discoveries depend on previous work which in turn guides further theory and experimentation; and (3) the reigning paradigms, i.e., ideas, theories, hypothesis, experimentation, are all guided by existing paradigms or scientific frameworks for guiding interpretations and confirmations (Kuhn 1962). The relative role of these factors of course varies between disciplines. 
The object of investigation can be an idea, an equation, invisible physical forces, energy, or objects -living or non-living - or materials in space and time. The material bases of science also vary in their degree of openness and accessibility in being fully, partly, or not at all accessible (Stent 1969). By “openness" I mean if a field which has no physical boundary in choosing its problem or objects of studies, and by "bounded" I mean a field which has real boundary and limit in choosing the objects of its studies. The material nature of the field and the degree of accessibility can be jointly used to characterize the different disciplines of science as shown in Table 1.

Mathematics is open-ended and this is especially true in view of Gödel's theorem of insufficiency of any given axiomatic system (To quote William Blake, "you never know what is enough unless you know what is more than enough"). Mathematics' domain is logic and it relates to private events arising from the inner world and its application to public events from the outside world; it has no boundary and therefore it can never be fully accessible. Physics is partly open in the sense that only part of the subject matter of physics - matter, energy, electromagnetic forces, light - are visible to our senses and the current state of knowledge does not guarantee that we know it all. The recent discovery of dark matter and dark energy is a case in point. Astronomy is bounded in the sense that it deals with physical objects - planets, stars, galaxies. It provides partial and of course limited access and that too is dependent on heavy instrumentation. Geology is bounded and it includes study of rocks, natural resources, water, and geological and weathering forces. Chemistry encompasses elements, minerals, and chemicals and we know enough about different classes of chemicals but again there is no guarantee that we know it all, especially if we include chemical interactions and the possibility that still much remains unknown. Biology deals with organisms and while some organisms may be invisible to the naked eye, they are all physically defined, distinct, and bounded in space and tine and are potentially fully accessible for study. This unique nature of biology, i.e., the objects of its study, the organisms being bounded in space and time and their accessibility- dictates how biological studies are planned and carried out. Organism is the black box of biology and biology has full access to it. 
Scientific discoveries depend on methodology. Conceptual breakthroughs are generally few and far in-between but they have of course major impacts when they do occur. Darwin's theory of natural selection revolutionized how we look at life - past, present and future. Serendipitous discoveries, such as the discovery of penicillin, can occur in any field of science. Theoretical modeling in combination with data can go a long way as was the case with Watson and Crick's modeling of DNA structure with the help of Rosalind Franklin's X-ray diffraction data on DNA.

Mendel's and McClintock's discoveries were unique in that they dealt with two sides of the same coin. Mendel's problem consisted in explaining heredity, i.e. how traits are inherited and how they remain invariant or stable over generations. In contrast, McClintock's problem consisted in explaining why some traits such as the Corn seed coat colors were hyper-variable or unstable? Mendel discovered laws of heredity without knowing what the underlying trait "factors" were made of, or how they segregated (Singh 2015). McClintock discovered mobile genetic elements without knowing the underlying molecular mechanism of generating variation. Both discoveries dealt with the same black box but with different inputs and outputs. Mendel's discovery went unappreciated because the focus at that time was on a different paradigm: role of hybrid development in breeding and in species creation. McClintock's discovery went unappreciated as it went against the reigning paradigm of the time - chromosomal basis of heredity and genetic linkage, genes having fixed positions on chromosomes. Her work became appreciated only after similar phenomena were discovered with micro-organisms.

\section{Science boundaries and the nature of discovery}

Beside origin of life, conceptually speaking, biology has four important questions to answer: How organisms develop from a single fertilized cell to a complex entity? How they function and maintain their physiological integrity and homeostasis? How they reproduce? And how they evolve? These are the important questions, respectively, of the field of developmental biology, physiology, genetics, and evolutionary biology. The majority (45\%) of the Nobel Prizes in Physiology or Medicine have been awarded, not unexpectedly, for experimental work based on 
structural -functional details of the organism (including vision, circulation, respiration, digestion, immunity, neurons, hearing, etc), $25 \%$ for research related to medicine, $13 \%$ for research on disease-related micro-organisms, and $13 \%$ for work related to genetic machinery/genetic transmission. Interestingly, in all of its 114 years of existence there is only one Nobel Prize awarded in the area of whole organism biology - the prize shared by Karl von Frisch, Konrad Lorenz, and Nicolas Tinbergen for their "discoveries concerning organization and elicitation of individual and social behavior patterns" in 1973. (This is not because there have been no worthy candidates in evolutionary biology. This says more about the initial criteria of what constitutes an important discovery than important contribution to the advancement of knowledge, Dobzhansky, Fisher, Haldane, Wright, Kimura, not to mention any living individuals, were all worthy candidates as they each made tremendous contribution to the advancement of evolutionary biology).

Thus with the exception of work that may be related to developing research tools (such as tomography, magnetic resonance imaging, mutation by X-ray radiation) and discovery of genetic structures (such as the DNA double helix, split genes, and mobile genetic elements) more and more of biological research in the future will be based on the inner working of the organisms' physiology as we dig deeper and deeper into tissues, cells, organelles, and genomes. This will leave less and less room for conceptual breakthroughs spanning different levels of organizations, fewer leaps beyond boundaries like those spanned by Mendel and McClintock.

As a comparison, in physics of the important discoveries (again based on award of Nobel Prizes, 1901-2014), nearly half (46\%) are based on theoretical -experimental elaboration of matter and forces, $20 \%$ on atomic-subatomic particles, $28 \%$ on instrumental $/$ methods development, and $6 \%$ on astrophysics and astronomy. Physics is dominated by theoretical and instrumental discovery and biology by experimental, and this means that premature discoveries in physics differ from those in biology. As examples I present a few cases of premature discovery in physics that provide a contrast from those in biology. 
Dark matter was postulated by Jan Oort in 1932 to account for the orbital velocities of stars in the Milky Way and first discovered by Fritz Zwicky in 1937 to account for evidence of "missing mass' in the orbital velocities of galaxies in clusters. However, it was not until thirty years later that robust evidence was produced by Vera Rubin in the 1960s-1970s, using single galaxy rotation curves.

The chaos theory got its real start in the study of dynamical systems and nonlinear differential equations (Birkoff 1927), in the study of turbulence and astronomical problems (Kolmogorov 1941), and in the study of radio engineering (Cartwright and Littlewood 1945). But it was not until twenty years later that evidence for chaotic behavior of dynamical systems came from meteorology by Edward Lorenz (1963) and from fluctuation of cotton prices (Mandelbrot 1963). The main catalyst for the development of the chaos theory came from electronic computer and the theory saw its application in a wide variety of fields including earthquakes, solar flares, financial markets, and even in evolution - by punctuated equilibrium (Eldredge and Gould 1972).

Gauss first looked at curved space and Riemann developed non-Euclidean geometry, a theory of curved space in the physical world but it was not a hot area of interest. It was ignored by physicists until Einstein used it to describe his famous curved-space-time theory in the theory of gravitation.

Another example is the discovery of light bending predicted by Einstein which was confirmed in 1919. In 1936 Einstein made calculations, on the request from an Engineer friend, and found that the light bending by a star was too small to be effective as lenses. Apparently it did not occurr to him that the same bending could be much larger by a larger mass like a galaxy, or a cluster of galaxies (Kraus 2015). More than 50 years later gravitational lensing has become the principal tool by which dark matter masses are detected.

There are also cases in physics where gender discrimination may have played a role. 
Henrietta Leavitt discovered that the absolute luminosity of "Cepheid variable" stars that wax and wane could be deduced from the observed period of waxing and waning. Measuring the apparent luminosity on earth, the star looked dimmer. Since the intensity of light falls off as the square of the distance, one could deduce the distance of the star from the earth. The Cepheid Variables could then be used as "standard candles". This discovery was crucial for the subsequent measurements of Hubble, and the progress of astronomy and cosmology. Henrietta died unrecognized for her work. Some say gender discrimination may have played a role as in the case of Rosalind Franklin in biology.

Another example is that of Lise Meitner (born in 1891 in Vienna) who became a professor at Kaiser Wilhelm Institute in Berlin and worked, with Otto Hahn, on decay of radioactive nuclei induced by slow neutrons. Meitner was a "non-Aryan" and had to flee Nazi Germany in 1938. Hahn published the results excluding Meitner and was awarded Nobel Prize in 1944. Meitner was neglected by the Nobel Committee despite multiple nominations by Niels Bohr (Sime 1996).

The first four examples are similar in starting as a theory and becoming widely recognized only after their importance and wide applications were discovered /demonstrated. The latter two are probable cases of gender discrimination not unlike that of Rosalind Franklin in Biology.

\section{Are premature discoveries things of the past?}

Science is a historical process and it progresses by feedback; the discoveries of today will impact those of tomorrow. There are several reasons why premature discoveries are less likely if not totally out. First, there are more scientists engaged in every branch of science and this alone would keep important discoveries under the community's radar. Second, more and more of science publications are becoming open, on-line, and interactive, and so the probability of an important discovery remaining buried and unrecognized would become smaller and smaller. Finally, in all branches of sciences major unsolved problems are on the front burners and known and as a result the lag time between major discovery and its recognition is progressively 
shrinking. This is especially true of serendipitous discovery but not of those based on major lifelong contribution to the progress of science which still may take longer time.

There is another reason why future discoveries in biology would not go un-noticed for long. Of the four major research areas of biology, origin of life, genetic information systems, development, physiology-function, and evolution, the most unknowns lie in the area of origin of life and development, and of these, development is obviously the focus of current scientific engagements. While we can never rule out discoveries of serendipitous nature or of those impacting the development of genetics tools, it is most likely that more and more of future discoveries will be based on details of cellular structure and function which will be known and celebrated not so much as novel discoveries as important contributions to the advancement of the field. Organisms are time-and- space-extended cells and so the laws of development will also be of distributed nature over space and time. As pointed out above, the majority of Nobel Prizes in biology are based on contribution to the advancement of the field and these kinds of discoveries are based on cumulative knowledge on a given problem and hence less likely to be ignored.

Of course, there would still remain a difference in the probability of premature discovery in different fields of science because of the difference in the accessibility of the field. Biological sciences, for example, are proceeding from outside in, becoming more and more genomicsbased. Furthermore there is a shift toward big science, involving massive data collection, and the hierarchical boundaries between cellular levels are disappearing fast. The nature of current biology making it increasingly dependent on large consortiums and collaborations will make it less likely that major, significant discoveries would go unrecognized for long. On the other hand, as the examples from physics show, a new theory or an important idea can always remain low key or even unknown until it becomes used and popularized by other researchers.

\section{Conclusion}


Evolution of science passes through four stages: First there is the conceptual -theoretical frame works which set the problems and which in the initial stages generally require taking leaps across the boundary of the known, preliminary knowledge. Second stage involves instrumentation, experimental verification, and accelerated expansion of knowledge that ultimately invite questions and concerns such as "do we know it all?", or limits of science. Third stage consists of doing science by pushing the boundary of knowledge mostly from inside and less by leaping across boundaries. Finally, truly innovative science may be done by going across the boundary of major disciplines such as biology and physics, biology and material science, biology and chemistry, and by mounting new rounds of experimentation and exploration. From modern standard Mendel would seem to have operated within boundary, but from the standard of his time he leaped across boundary. History shows that chances of premature discoveries are more when progress in science is being made by leaping across boundaries than by pushing science incrementally from inside the boundary. Modern sciences are reaching their respective boundaries and science is under watch twenty four hours a day by hundreds of thousands of scientists working and watching online from all over the world. It's less likely that a major discovery would go unnoticed for long. Premature discoveries will become less likely but not forbidden.

\section{Acknowledgement}

I am most grateful to Professor Freeman Dyson and Rajat Bhaduri for bringing cases of premature discoveries in physics to my attention, and to Richard Lewontin, Daniel Hartl and Richard Morton for their comments on the manuscript. This work was supported by a grant from the Natural Sciences and Engineering Research Council of Canada (NSERC). The author has no conflict of interest to declare. 


\section{References}

Birkoff, G.D. 1927. Dynamical Systems. Vol 9 of the American Mathematical Society Colloquium Publications. Providence, Rhode Island: American Mathematical Society)

Cartwright, M.L. and J.E. Little wood. 1945. On non-linear differential equations of the second order. Journal of the London Mathematical Society 20 (3):180-189.

Eldredge, N., and S. J. Gould.1972. Punctuated equilibria: An alternative to phyletic gradualism. In. T.J.M. Schopf, ed., Models in Paleobiology. San Francisco: Freeman, Cooper \& Co., pp.82115.

Hartl, D. L., and D. J. Fairbanks. 2007. Mud sticks: On the alleged falsification of Mendel's data. Genetics 175: 975-979.

Kolmogorov, A. N. 1941. The local structure of turbulence in an incompressible viscous fluid for very large Reynolds numbers. Proceedings of the Royal Society A 434 (1890): 9-13.

Krauss, M. 2015. What Einstein got wrong. In Scientific American, September, 2015. Page 51.

Kuhn, T.S. 1962. The Structure of Scientific Revolutions. Chicago: The University of Chicago Press.

Kuhn, T. S. 1974. The Essential Tension: Selected Studies in the Scientific Traditions and Change. Chicago: The University of Chicago Press.

Lorenz, E. 1963. Deterministic non-periodic flow. Journal of the Atmospheric Sciences 20(2): $130-141$.

Mandelbrot, B. 1963. The variation of certain speculative prices. Journal of business 36 (4): 394-419.

Mendel, G. 1866. Versuche uber Pflanzenhybriden. Verh. Naturforsch. Ver. Brunn, 4: 3-47. Translation: in Bateson, W. 1909, 1913. Mendel's Principles of Heredity???

McClintock, Barbara. 1984. A Feeling for the Organism, 10th Anniversary Edition: The Life and Work of Barbara McClintock 10 Anv. Edition by Keller, Evelyn Fox. Times Books (Paperback).

Sime, R.L. 1996. Biography of Lise Meitner. Berkley, CA: University of California Press.

Singh, R. S. 2015. Limits of imaginations: The $150^{\text {th }}$ anniversary of Mendel's Laws and why Mendel failed to see the importance of his own discovery for Darwin's theory of evolution. DOI $10.1139 /$ gen-2015-0107 
Stent, G. 1969. The Coming of the Golden Age: A View of the End of Progress. New York: The American Museum of Natural History, The Natural History Press.

Sturtevant, A. H. 1965. A History of Genetics. Cold Spring Harbor: Cold Spring Harbor Laboratory Press. 
Singh, Rama: Science Beyond boundary: Are premature discoveries things of the past?

Table 1. Science Boundaries \& field accessibility

Mathematics: Open, partly accessible

Physics: Partly open, partly accessible

Chemistry: $\quad$ Partly open, partly accessible

Astronomy: Bounded, partly accessible

Geology: $\quad$ Bounded, partly accessible

Biology: $\quad$ Bounded, fully accessible 